3 Research Square

\title{
Evaluation of Intravitreal Bevacizumab injection in treatment of diabetic macular edema in eyes with Good visual acuity
}

seyed aliasghar mosavi ( $\sim$ a.a.mosavi@gmail.com )

Research article

Keywords: Diabetic macular edema, Bevacizumab, Anti VEGF, Good vision

Posted Date: July 16th, 2021

DOI: https://doi.org/10.21203/rs.3.rs-36672/v1

License: (c) (i) This work is licensed under a Creative Commons Attribution 4.0 International License.

Read Full License 


\section{Abstract \\ Aims}

To determine the effect of intravitreous Bevacizumab injection on reducing diabetic macular edema in patients with good vision.

\section{Method}

: This clinical trial was conducted on diabetic patients with centrally involved macular edema and good vision over a period of one and a half year. 22 eyes of 12 patients with a visual acuity of Snellen equivalent of $20 / 25$ or better in the non-proliferative stage with the central macular thickness of more than 300 microns in optical coherence tomography (OCT) were evaluated. In the injection group $1.25 \mathrm{Mg}$ of bevacizumab (Avastin) was injected intravitreally and the control group was followed up. Macular thickness and visual acuity were measured in two groups at first and sixth months after injection.

\section{Results}

Intravitreal bevacizumab was injected in 12 eyes (55\%) and 10 patients were followed-up as a control group (45\%). The mean central macular thickness was $319 \mu \mathrm{m}$ at first visit and $301 \mu \mathrm{m}$ at month six in the treatment group and in the control group it was $318 \mu \mathrm{m}$ and $328 \mu \mathrm{m}$ respectively. The mean macular thickness of the treatment group was significantly decreased $(p=0.031)$, but the difference was not statistically significant between two groups $(p=0.581)$. The mean visual acuity in the treatment group was 0.045 Log MAR at first visit and 0.033 Log MAR at the sixth month follow up, these values in the control group were 0.1 Log MAR and 0.045 Log MAR respectively and did not differ statistically between the two groups $(p=0.373)$

\section{Conclusion}

Although the mean macular thickness was reduced in the treatment group with visual acuity of 20/25 or better but the difference was not significant during follow-up at least in a short period. Therefore, the role of underlying factors other than VEGF alone can be notable in the pathogenesis of the disease.

\section{Introduction}

Diabetic retinopathy is the fifth leading cause of blindness worldwide and the most important preventable cause of vision loss in people of working age (20-74 years) in developed countries. [1] By 2040, it is projected that the number of people with diabetes will exceed 600 million. Epidemiological studies show that one out of every 3 people with diabetes has diabetic retinopathy and one out of every 10 diabetic people have macular edema and Vision-impairing complications [2, 3] .Diabetic Macular Edema can 
develop at any stage of the disease, although it will be absorbed in half of the untreated cases but reduces central vision by 15 letters or two lines in Snellen's visual acuity chart within two years. [4] Although the pathogenesis of diabetic macular edema is not yet fully understood, it seems that vascular endothelial growth factor (VEGF) secreted by hypoxia due to microvascular complications of diabetes has the greatest effect on retinal tissue. VEGF is a very important mediator in damaging the retinal blood barrier and by damaging this barrier will increase the permeability of vessels and macromolecules to the liquid and macromolecules from the retina choriocapillaries and extracellular fluid accumulation, leading to dysfunction and eventually disruption of retinal layers $[5,6]$ Nowadays, due to different levels of response in different patients and some cases of refractory macular edema and more attention to personalization treatment, molecular studies and analyzes of aqueous humor proteins have been performed. New therapeutic theories and targets have been proposed, including counteracting TI2 inhibitors, which is a vascular endothelial stabilizing protein [7]. The creation of advanced imaging techniques, including optical coherence tomography (OCT), is now possible through the use of optical reflection, the creation of dimensional images, and the determination of the thickness of the retinal layers, and the detection of small amounts of edema in the macula that is the cornerstone of edema detection. [8-10] Given the possibility of early detection of diabetic macular edema (DME) and the potential risk of vision loss in patients, it is believed that patients will benefit from early treatment of macular edema, and various treatments for DME have been used over the past three decades, including laser macula photocoagulation, intravitreous injection and combination therapy. New clinical trials in patients with macular edema indicate that patients receiving anti-VEGF injections have better vision than those who did not receive treatment. They have less tissue degradation and therefore have better efficacy and fewer side effects and are currently being used with strong evidence as the first line of treatment in the world [11].

Bevacizumab (Avastin) is a recombinant monoclonal antibody that inhibits all VEGF isoforms that was approved by the American Food and Drug Administration (FDA) and European Medicines Agency (EMA). [6] Early treatment can be effective in reducing vision but injecting the drug into the vitreous requires sterile operating room conditions, in addition to imposing costs on the patient that may lead to double stress. On the other hand, the number of prospective studies to guide management of eyes with diabetic macular edema with good VA is very limited, so we decided in this study to evaluate the efficacy of Anti VEGF (Bevacizumab) treatment in DME patients with good vision.

\section{Methods}

This prospective, consecutive, interventional study included patients with clinically significant diabetic macular edema who referred to the Ophthalmology Clinic at Bina Eye Hospital (Tehran, Iran). Patients were only evaluated if they were willing to participate, with visual acuity of $20 / 25$ or better, age $>=18$, non-proliferative stage of diabetic retinopathy, central macular thickness of more than 300 microns in optical coherence tomography and patients that had been treated with at least 3 anti-VEGF intravitreal injections during this period. If both eyes of a patient were eligible, the eye that had received the greater number of anti-VEGF injections was designated as the study eye. Patients were excluded if they had 
uncontrolled hypertension, a history of myocardial infarction or stroke in the last 3 months (considering the side effects of systemic Avastin), or any types of medial opacity, e.g. cataract, or vitreous hemorrhage, due to drug injection or due to a background disease. All eligible patients with DME were included and provided with explanations about the method and probable adverse effects and results of injection and also other methods of therapy and their results. The patients then signed an informed consent. Before injection, a full ophthalmic examination was performed by an ophthalmologist (A.A) and the results as well as the individual data were recorded in special forms. Fundus photography and optical coherence tomography (OCT) were conducted for each patient and the results were recorded. Visual acuity was measured with standard Snellen charts, then converted to Log MAR measurements for ease of calculation. Clinically significant macular edema was diagnosed using slit lamp ophthalmoscopy with a noncontact $78 \mathrm{D}$ lens upon the standard definition. Spectralis OCT (Heidelberg Engineering, Germany) was performed for all patients. The study was based on Helsinki's principles, and ethical approval was obtained by the Ethics Committee in Human Research at Baqiyatallah University of Medical Sciences (registered number of IR. BMSU004). Preparing ampoules from the vials was performed under sterile conditions. Avastin was made in Gentec Company (USA). In the operating room, the patient was prepared for intravitreal injection. Prepping and draping were done with Betadine $10 \%$. In addition, a single drop of Betadine was instilled in the lower fornix and after 5 minutes, the fornices and the ocular surface were irrigated with balanced salt solution (BSS). A topical sterile anesthetic (tetracaine $5 \%$ ) was then applied. The lids were kept open with a lid speculum. Afterwards, $0.05 \mathrm{ml}(1.25 \mathrm{mg})$ of the Avastin solution $(10 \mathrm{mg} / \mathrm{ml}$ ) was injected into the vitreous body at the pars plana (the distance from limbus was $4 \mathrm{~mm}$ in phakic and $3 \mathrm{~mm}$ in aphakic or psuedophakic patients). The amount of the injected drug has been selected according to previous similar studies. After manual checking, if the intraocular pressure was high, fluid tap, with an amount less than or equal to the injected volume, was performed from the anterior chamber. A single drop of topical antibiotic was then instilled and the eye was patched. The patients were prescribed with the antibiotics drops for 7 days and discharged. The patients were visited on the next day and after 1 week. They were advised to refer quickly to the emergency room in case of symptoms such as pain, redness, and vision loss. Those who were assigned to intravitreous avastin received injections every 4weeks as needed depending on visual acuity and OCT-measured retinal thickness. In addition, at least 2 more injections were performed with a similar method (resulting in a total of 3 injections) and the examination results in the $1 \mathrm{st}, 2 \mathrm{nd}$ and 6 th months were recorded. After the completion of the examination forms, the collected data was analyzed in SPSS20 (SPSS Inc., Chicago, IL, USA)

\section{Results}

This was a prospective clinical trial on 22 eyes of 12 patients including 7 males ( 12 eyes) and 5 females (10 eyes). The intravitreous avastin was injected at least three injections with every 4 weeks interval as needed depending on visual acuity and OCT-measured retinal thickness. The mean age of participants was $63 \pm 5$ years (range: $48-70$ years). 12 patients were injected (55\%) and 10 patients were in the control group (45\%). The mean of macular thickness before injection was $319 \mu \mathrm{m}$ and $301 \mu \mathrm{m}$ at month six in the treatment group and in the control group 318 and $328 \mu \mathrm{m}$, respectively. The mean macular 
thickness of the treatment group was significantly decreased $(p=0.031)$, but the difference was not statistically significant between two groups $(p=0.581)$. The mean visual acuity in the treatment group was 0.045 Log MAR at first visit and 0.033 Log MAR at the sixth month follow up, these values in the control group were 0.1 Log MAR and 0.045 Log MAR respectively and did not differ statistically between the two groups $(p=0.373$,). (Figs. 1 and 2$)$.

\section{Discussion}

DME remains the most common cause of reduced vision in diabetic patients that puts the Visual acuity at risk. Angiogenesis inhibitors are widely used throughout the world today as the first line of treatment for diabetic macular edema $[3,18]$, and these drugs have the potential to enhance the preservation of vision and even increase vision in many patients. In this survey, we assessed the effects of Avastin on macular thickness in eyes with DME and good visual acuity. The results of this clinical trial showed that although the mean central macular thickness was decreased in the treatment group, it was not significantly different from the control group and there was no significant difference in the two groups.

The pathophysiology of diabetic edema can determine the individual patient's medical need, which may increase angiogenesis in one group and inflammatory factors in the other, or both. Disorders are vascular permeability and therefore each will need its own unique treatment [7]. Systematic review studies conducted by the Cochrane Library with high evidence have found the use of antiangiogenic drugs to be effective in the treatment of macular edema [11, 18], while research by the American Diabetes Association demonstrated resistant to this drug with no clinical response in approximately $40 \%$ of patients [19]. In recent years, several studies have investigated the efficacy and comparability of different groups of angiogenic drugs and have evaluated their therapeutic results with other available therapies such as laser and corticosteroid therapy. Given the presence of resistant cases, underlying factors beyond VEGF may play a role in the pathogenesis of the disease, and the investigation of molecular factors and changes in intraocular protein levels in response to treatment and genetic studies may offer a new approach to treatment [10] So,there seems to be a ceiling for treatment with antiangiogenics, and then no further treatment.

Study by Baker, C.W., et al [ 12 ] in a randomized clinical trial in 702 participants with diabetic macular edema involving the center of the macula (CI-DME) and good visual acuity that were evaluated in term of the effect of initial management with aflibercept vs laser photocoagulation vs observation on vision loss, no significant difference in vision loss at 2 years was found whether eyes were initially managed with aflibercept or with laser photocoagulation or observation and given aflibercept only if visual acuity worsened. They concluded observation without treatment unless visual acuity worsens may be a reasonable strategy for Cl-DME. Also, in a retrospective study Busch, C. et al. [13] reported evaluation of baseline predictors for visual acuity loss during observation in diabetic macular edema with good baseline visual acuity in 249 eyes of which 147 eyes were observed and 80 eyes received anti-vascular endothelial growth factor (VEGF) treatment. They found that earlier treatment with anti-VEGF in these patients may potentially decrease the risk of VA loss at 12 months. 
Another study by Luu, K.Y. et al. [ 14] on 122 eyes of 100 patients with treatment-naive DMO and initial best-corrected visual acuity (BCVA) of $20 / 25$ or better that were received cumulative intravitreal injections and laser treatments at yearly intervals. They revealed DMO with good initial visual acuity should be monitored closely, as delay in treatment initiation is associated with worse visual outcomes and concluded BCVA at time of initial treatment is the strongest determinant of final visual acuity.

On one hand our results are in contrast to the study by Busch, C.et al that believe Earlier treatment with anti-VEGF in these patients may potentially decrease the risk of VA loss. On the other hand, despite the small sample of eyes in our study, our finding is similar to the study of Baker, C.W., and Luu, K.Y. that suggest closely monitoring of subjects with DME

A systematic review study by Pravin U Dugel et al., investigating the association between baseline (pretreatment) vision and increased visual acuity in patients treated with anti-angiogenesis drugs for an average of 12 months, they stated "The amount of vision corrected before treatment is inversely correlated with the rate of increased vision after treatment." This study, based on 9 major studies :RESOLVE, RISE, RIDE, RESTORE, RETAIN, RETAIN, DRCR.net Protocol I, VINCI, VIVID VISTA, suggests that no matter the amount of anti-angiogenic drug used and the theoretical coincidence, among all of them there is a steady increase in visual acuity of more than $20 / 40$ and then a slight increase in visual acuity afterwards. [17] that is in line with the results of our study.

As reported in previous study using angiography, there is a blood vessel-free zone in the fovea region of diabetic patients due to hypoxia, suggesting that macular edema may play a different mechanism. Edema from other regions of the retina not affected by the production of angiogenic factors and in relation to the failure of the retinal pigment epithelial cell pump due to choriocapillary hypoxia may explain the lack of association between non-reduction of retinal thickness using anti-angiogenesis drugs [10]. In addition, Browning et al. stated in their review study that the results of numerous studies around the world in terms of the average number of intravitreal injections and increased visibility with renowned clinical trials is a significant difference [15.16] which believe that in the real world the use of antiangiogenic drugs accompany multiple visits and time expenses. Furthermore, according to a previous clinical trial report [10] diabetes is a nasty disease that yield a permanent photoreceptor damage based on the duration of disease or from extensive previous treatments. It may also be attributable to individual systemic factors that may affect macular edema such as type of diabetes and glycemic control of diabetes, age, blood pressure, serum lipid levels, and nephropathy. In addition, it is not known whether or not continued injections for six to 12 months could improve the outcomes. So we should not consider simple mechanisms for its complications which if given adequate health care and in-vitro antiangiogenesis injections, will accompany high rates of recovery .

Our study has potential limitations, including the small sample of treated eyes, and slightly short term follow ups. However, the results in our study are similar to those in DME study in which closely monitoring were suggested for treatment. 
In conclusion, we have shown that although Avastin may be useful for reducing the incidence of diabetic macular edema in patients with visual acuity of $20 / 25$ or better at least in a short period., it seems that, its effect is temporary and is not significant compared to the control group .However, further studies with a longer follow-up period and a larger number of patients are recommended to draw final conclusions about the efficacy of Avastin and their role in controlling the progression of DME

\section{Declarations}

\section{Acknowledgements}

The authors would like to acknowledge the staffs of Eye Clinic in Bina Eye Hospital for their valuable help during the entire process of this study.

\section{Consent for publication}

Not Applicable

\section{Funding}

The whole study was performed without any funding.

\section{Availability of data and materials}

The datasets generated and/or analyzed during the current study are not publicly available since all relevant data are included in the manuscript. The datasets are available from the corresponding author on reasonable request.

\section{Authors' contributions}

$\mathrm{AA}, \mathrm{HT}$ and $\mathrm{NI}$ were responsible for the conception and design of the study. $\mathrm{NI}$ acquired the data. AM;AA, and $A A$ analyzed and interpreted the data. SAM wrote the draft. AA, HTand AA revised the manuscript critically. All authors have read and approved the final manuscript.

\section{Ethics approval and consent to participate}

The research protocol was in accordance with the Helsinki's Declaration and approved by the Ethics Committee in Human Research at Bina Eye Hospital , Tehran (registered number of IR. BMSU004).

\section{Competing interests}

The authors declare that they have no competing interests.

\section{Author details}

${ }^{1}$ Department of Ophthalmology, Hormozgan University of medical sciences, Bandar Abbas, Iran 
${ }^{2}$ Vision Health Research Center, Semnan University of Medical Sciences, Semnan, Iran

${ }^{3}$ Health Management Research Center, Baqiyatallah University of Medical Sciences, Tehran, Iran

\section{Abbreviations}

Optical coherence tomography (OCT) ; Best-Corrected Visual Acuity(BCVA); diabetic macular edema (DME); American Food and Drug Administration (FDA) ; European Medicines Agency (EMA); vascular endothelial growth factor (VEGF).

\section{References}

1. Stefanini FR, Arevalo JF, Maia M. Bevacizumab for the management of diabetic macular edema. World J Diabetes. 2013;4(2):19.

2. Lee R, Wong TY, Sabanayagam C. Epidemiology of diabetic retinopathy, diabetic macular edema and related vision loss. EYE VISION. 2015;2(1):17.

3. Wong TY, Sun J, Kawasaki R, Ruamviboonsuk P, Gupta N, Lansingh VC, Maia M, Mathenge W, Moreker S, Muqit MM, Resnikoff. S. Guidelines on diabetic eye care: the international council of ophthalmology recommendations for screening, follow-up, referral, and treatment based on resource settings. J Ophthalmol. 2018;125(10):1608-22.

4. Edington M, Sachdev A, Morjaria R, Chong V. STRUCTURAL-FUNCTIONAL CORRELATION IN PATIENTS WITH DIABETIC MACULAR EDEMA. Retina. 2017;37(5):881-5.

5. Ferris FL III, Patz A. Macular edema. A complication of diabetic retinopathy. Surv Ophthalmol. 1984;28:452-61.

6. Gerendas, B.S.

Schmidt-Erfurth U, Garcia-Arumi J, Bandello F, Berg K, Chakravarthy U. Gerendas, B.S.

7. Jonas J, Larsen M, Tadayoni R, Loewenstein A. Guidelines for the management of diabetic macular edema by the European Society of Retina Specialists (EURETINA). Ophthalmologica. 2017;237(4):185-222.

8. Jonas JB, Jonas RA, Neumaier M, Findeisen P. Cytokine concentration in aqueous humor of eyes with diabetic macular edema. Retina. 2012;32(10):2150-7.

9. Virgili G, Menchini F, Casazza G, Hogg R, Das RR, Wang X, Michelessi M, Optical coherence tomography (OCT) for detection of macular oedema in patients with diabetic retinopathy. Cochrane Database of Systematic Reviews, (1). 2015.

10. Hirano $T$, lesato $Y$, Toriyama $Y$, Imai A, Murata T. Detection of fovea-threatening diabetic macular edema by optical coherence tomography to maintain good vision by prophylactic treatment. OPHTHALMIC RES. 2014;52(2):65-73.

11. Browning DJ, Stewart MW, Lee C. Diabetic macular edema: evidence-based management. INDIAN J OPHTHALMOL. 2018;66(12):1736. 
12. Virgili G, Parravano M, Menchini F, Evans JR. Anti-vascular endothelial growth factor for diabetic macular oedema. Cochrane Database of Systematic Reviews, (10).2014.

13. Baker CW, Glassman AR, Beaulieu WT, Antoszyk AN, Browning DJ, Chalam KV, Grover S, Jampol LM, Jhaveri CD, Melia M, Stockdale CR. Effect of initial management with aflibercept vs laser photocoagulation vs observation on vision loss among patients with diabetic macular edema involving the center of the macula and good visual acuity: a randomized clinical trial. Jama. 2019;321(19):1880-94.

14. Busch C, Okada M, Zur D, Fraser-Bell S, Rodríguez-Valdés PJ, Cebeci Z, Lupidi M, Fung AT, Gabrielle $\mathrm{PH}$, Giancipoli E, Chaikitmongkol V. Baseline predictors for visual acuity loss during observation in diabetic macular oedema with good baseline visual acuity. Acta Ophthalmologica.2020.

15. Luu KY, Akhter MM, Durbin-Johnson BP, Moshiri A, Tran S, Morse LS, Park SS, Yiu G. 2019. Real-world management and long-term outcomes of diabetic macular oedema with good visual acuity. Eye, 2019 ,pp. 1-8.

16. Do DV, Nguyen QD, Shah SM, Browning DJ, Haller JA, Chu K, Yang K, Cedarbaum JM, Vitti RL, Ingerman A, Campochiaro PA. An exploratory study of the safety, tolerability and bioactivity of a single intravitreal injection of vascular endothelial growth factor Trap-Eye in patients with diabetic macular oedema. BRIT J OPHTHALMOL. 2009;93(2):144-9.

17. Aiello LP, Beck RW, Bressler NM, Browning DJ, Chalam KV, Davis M, Ferris III, Glassman FL, Maturi AR, Stockdale RK, Topping CR, T.M.. Rationale for the diabetic retinopathy clinical research network treatment protocol for center-involved diabetic macular edema. Ophthalmology,2011, 118(12), pp.e5e14.

18. Dugel PU, Hillenkamp J, Sivaprasad S, Vögeler J, Mousseau MC, Wenzel A, Margaron P, Hashmonay $\mathrm{R}$, Massin P. Baseline visual acuity strongly predicts visual acuity gain in patients with diabetic macular edema following anti-vascular endothelial growth factor treatment across trials. Clin Ophthalmol (Auckland NZ). 2016;10:1103.

19. Arevalo JF, Fromow-Guerra J, Quiroz-Mercado H, Sanchez JG, Wu L, Maia M, Berrocal MH, SolisVivanco A, Farah ME, Pan-American Collaborative Retina Study Group. Primary intravitreal bevacizumab (Avastin) for diabetic macular edema: results from the Pan-American Collaborative Retina Study Group at 6-month follow-up. Ophthalmology. 2007;114(4):743-50.

20. Haritoglou C, Kook D, Neubauer A, Wolf A, Priglinger S, Strauss R, Gandorfer A, Ulbig M, Kampik A. Intravitreal bevacizumab (Avastin) therapy for persistent diffuse diabetic macular edema. Retina,2006, 26(9), pp. 999-1005.

\section{Figures}




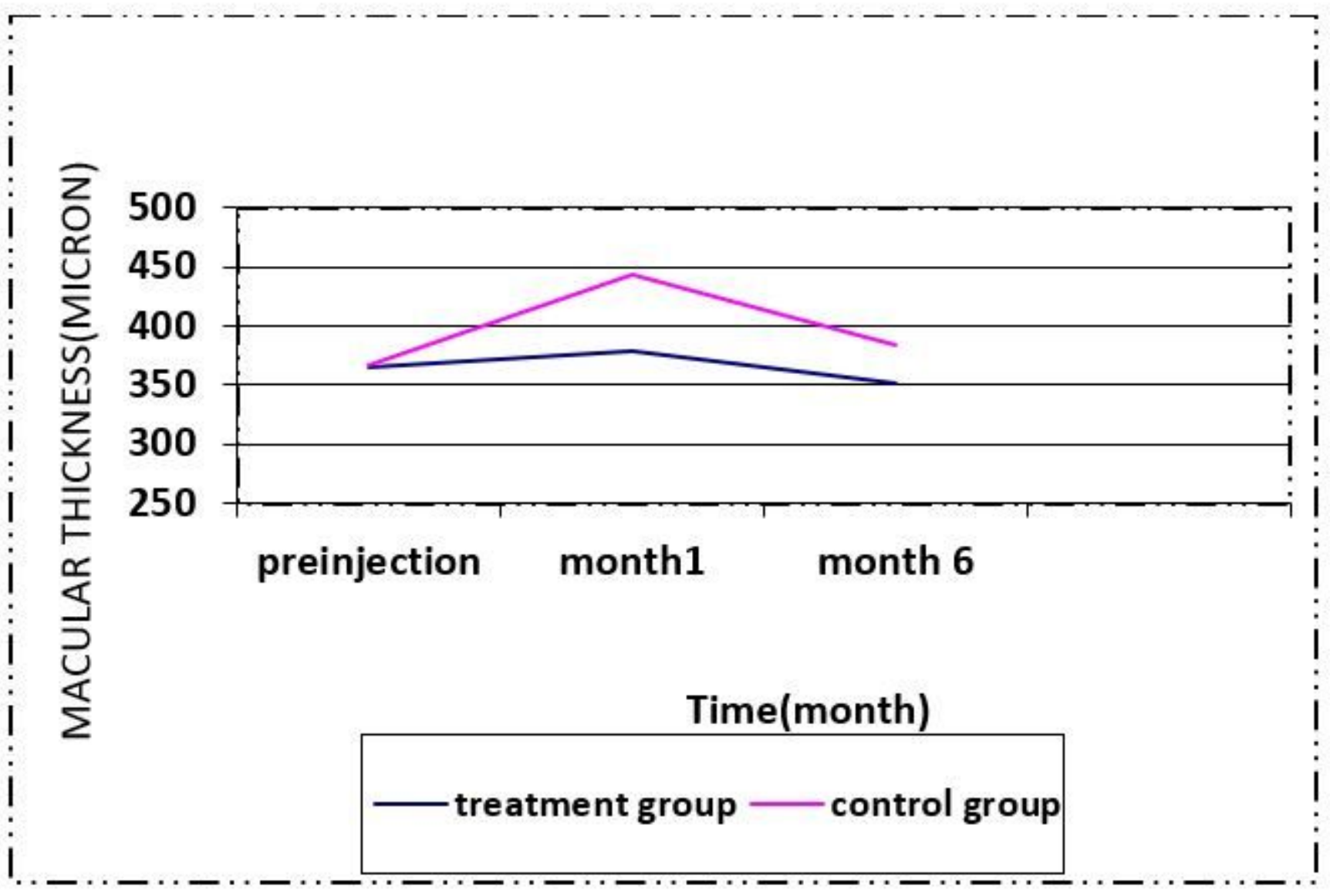

Figure 1

Mean change in macular thickness 


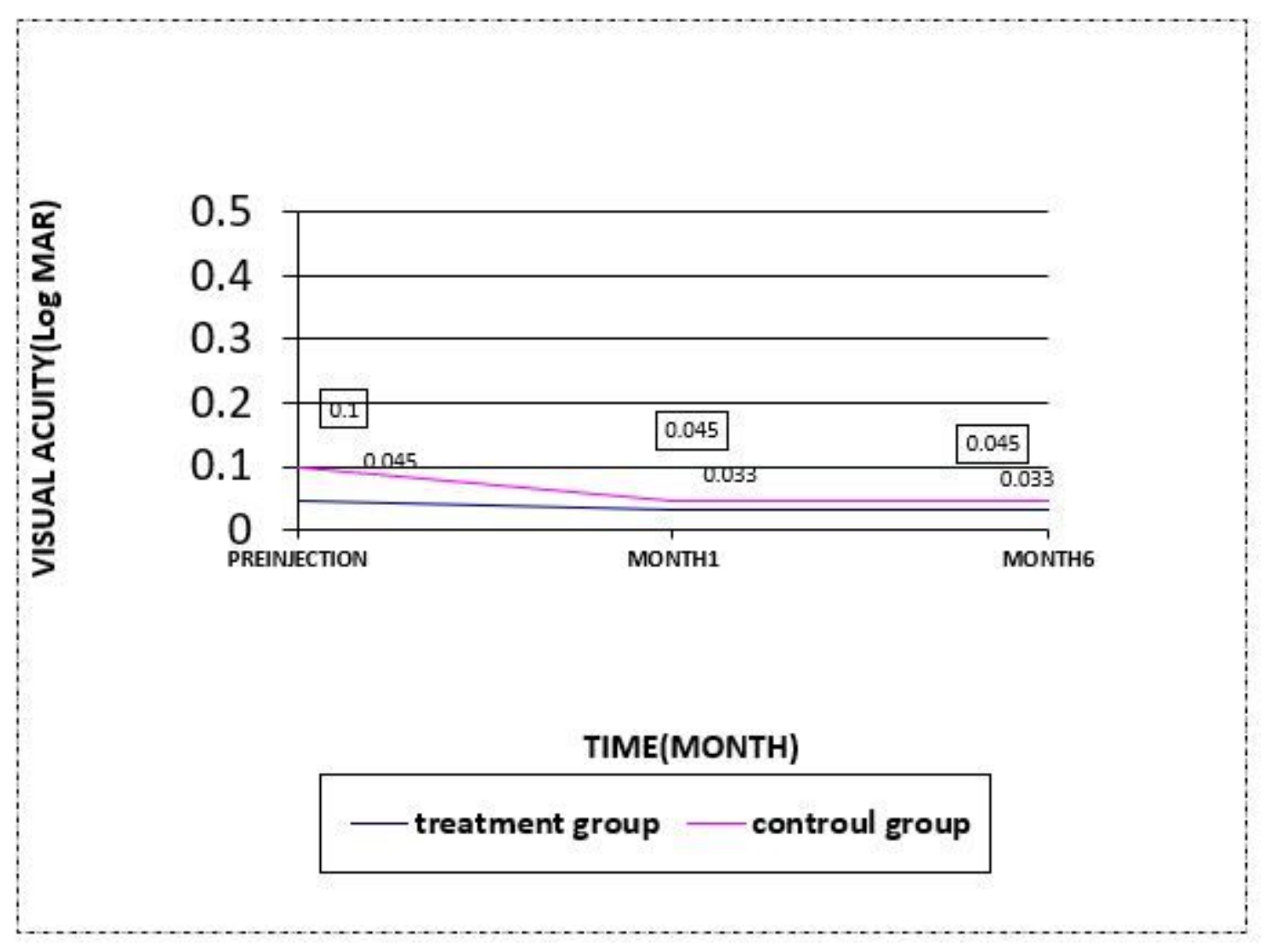

Figure 2

Mean change in visual acuity. 